\title{
Osteonecrosis Mandibular y Maxilar Secundaria a Bifosfonatos. Presentación de Tres Nuevos Casos
}

\author{
Mandibular and Maxilar Osteonecrosis Secondary to Bisphosphonates. \\ Introduction of Three New Cases
}

"Emilio González Jiménez; " Judit Álvarez Ferre \& *"* María José Aguilar Cordero

GONZÁlEZ, J. E.; ÁLVAREZ, F. J. \& AGUILAR C. M. J. Osteonecrosis mandibular y maxilar secundaria a bifosfonatos. Presentación de tres nuevos casos. Int. J. Morphol., 29(3):1022-1027, 2011.

RESUMEN: El empleo de los bifosfonatos supone en la actualidad una herramienta de especial relevancia para el manejo terapéutico de diversas patologías, en tanto que forman parte del tratamiento estándar. Sin embargo, y de un modo casi paralelo, se han documentado un amplio número de casos en los que el paciente, secuencialmente a su tratamiento con bifosfonatos, ha desarrollado un cuadro de necrosis avascular en región maxilar y/o mandibular. Cuadro éste, de complejo abordaje, que tiende a comprometer y mermar la calidad de vida de nuestros pacientes. No obstante, la posible aparición de esta complicación, no debe por otra parte, implicar reticencias en cuanto a su uso en la terapéutica, sino más bien constituir un aspecto a vigilar durante el tiempo de terapia. El objetivo de este trabajo es alertar sobre las posibles complicaciones en pacientes que reciben terapia con bifosfonatos a través de la revisión de la literatura y la presentación de tres nuevos casos clínicos.

PALABRAS CLAVE: Bifosfonatos; Osteonecrosis; Cáncer.

\section{INTRODUCCIÓN}

La necrosis avascular en regiones mandibular y maxilar, fue descrita por primera vez por Marx (2003). Su elevada prevalencia en los últimos años, ha supuesto una señal de alarma entre la comunidad científica y colectivo sanitario, dado el considerable número de casos descritos (Bagan et al., 2006).

Generalmente, la aparición de dicho cuadro, ha venido asociada al empleo de bifosfonatos tales como el pamidronato y ácido zoledrónico, sendos utilizados en el tratamiento y manejo de diversas patologías malignas como el mieloma, cáncer de testículo, de pulmón, de próstata, en sarcomas uterinos, en leucemia mieloide crónica postransplante de precursores hematopoyéticos y en pacientes con cáncer de mama en estadio metastásico, siendo este último, el grupo al que pertenecen los dos casos que presentamos (Lenz et al., 2005).

El mieloma y el cáncer de mama resultan ser las dos entidades clínico-patológicas que con mayor frecuencia y paralelamente a su tratamiento con bifosfonatos, llevan asociada la aparición de dicha patología (Durie et al., 2005a). Quizá este hecho deba su existencia al preponderante uso de estos agentes para el manejo terapéutico de las metástasis óseas, frecuentes en este tipo de tumores malignos.

Sin embargo, a pesar del marcado carácter sistémico de dicho cuadro, resulta llamativo lo selectivo del mismo en cuanto a afectación ósea. Son dos, las regiones óseas sobre las que se han descrito casos de necrosis avascular asociada a bifosfonatos. El maxilar superior, con una prevalencia de entre el $14-38 \%$ de casos (Vitte et al., 1996; Thakkar et al., 2006), y la mandíbula, en un $63-80 \%$ de pacientes. Tan sólo en un $9 \%$ de casos han sido descritas lesiones de este tipo en sendas localizaciones (Vitté et al., 1996; Durie et al., 2005b). Esta divergencia en cuanto a localización no resulta ser aleatoria, sino más bien responde a las características anatómicas y de modelado óseo de la zona. Las regiones maxilar y mandibular son áreas de pobre irrigación. Esto, unido a las características de la región bucal en cuanto

\footnotetext{
* Departamento de Enfermería. Facultad de Ciencias de la Salud. Universidad de Granada. Granada. España.

** Hospital Universitario "San Rafael”. Unidad de Hospitalización crónica. Granada, España.

**** Departamento de Enfermería. Facultad de Ciencias de la Salud. Universidad de Granada. Granada. España.
} 
a exposición a traumas e infecciones, hace de esta estructura anatómica un campo sin igual para la instauración y desarrollo de procesos necróticos avasculaes (Marx et al., 2005; Gutta \& Louis, 2007; Guirado et al., 2009).

Los maxilares y mandíbula comportan una tasa de actividad metabólica mayor a la del resto de los huesos. Este hecho, justifica el especial tropismo de las moléculas de bifosfonato por estas localizaciones óseas. Así, se ha podido comprobar, como la concentración de estos agentes es relativamente mayor en maxilar y mandíbula frente al resto de estructuras óseas corporales (Coleman et al., 2008).

Su potencial antiangiogénico, con la consiguiente disminución del factor de crecimiento endotelial y una vida media larga en las localizaciones descritas, ha determinado el cuestionamiento científico sobre su posible implicación en dicho proceso avascular necrótico (Dimitrakopoulos et al., 2006). Ahora bien, estudios de muestras histológicas de pacientes con osteonecrosis maxilo-mandibular asociada al empleo de terapia con bifosfonatos, revelan la existencia en ellas, de estructuras vasculares así como una vasculatura normal en tejidos adyacentes (Naveau \& Naveau, 2006; Lipton et al., 2008), lo que cuestiona en cierto modo la hipótesis comentada anteriormente.

Si parece resultar concluyente sin embargo, el efecto tóxico de los bifosfonatos, por dosis acumulada en dicho cuadro. Según Santini et al. (2002), la prevalencia del cuadro asciende según aumenta el tiempo de administración y dosis acumulada.

Luego a pesar de los continuos estudios realizados sobre este campo, carecemos de una visión certera del mecanismo exacto por el que se engendra y desarrolla esta entidad clínica. Conocemos los mecanismos que se suceden durante el metabolismo de estas sustancias pero son múltiples las incognitas que quedan por despejar en relación a su actividad. El estudio histológico y bioquímico minucioso constituye la vía de acceso y el campo de análisis para mejorar nuestro conocimiento al respecto.

Fundamentos bioquímicos de la terapia con bifosfonatos:

Para su correcto manejo terapéutico, resulta esencial mantener un preciso conocimiento acerca de los procesos que durante su metabolismo se suceden a nivel celular así como los diferentes mecanismos por los que estas moléculas ejercen su acción.

Los bifosfonatos, constituyen análogos químicos del pirofosfato inorgánico (Rogers, 2004). La molécula de pirofosfato inorgánico, se compone de dos átomos de fósforo y uno de oxígeno $(\mathrm{P}-\mathrm{O}-\mathrm{P})$.

Dicha molécula, presente en el plasma y líquido extracelular tiene como función la inhibición del depósito de minerales en la matriz orgánica de los tejidos. El osteoblasto, a su vez genera la enzima fosfatasa alcalina, cuya misión no será otra que degradar el pirofosfato inorgánico, permitiendo así el depósito de minerales tan necesario para la matriz ósea.

Los bifosfonatos, por su parte, difieren en su estructura química con el pirofosfato. Éstos, a diferencia del anterior, presentan dos átomos de fósforo unidos entre sí por un átomo de carbono $(\mathrm{P}-\mathrm{C}-\mathrm{P})$, dotando a este enlace de una mayor resistencia frente a la acción enzimática de la fosfatasa. Además, sus dos enlaces libres le confieren la posibilidad de concatenarse con cadenas laterales, lo cual, unido a la presencia de su grupo hidroxilo, determina la gran afinidad de esta molécula por los cristales de hidroxiapatita que conforman la estructura de nuestros huesos.

Luego, la acción de los bifosfonatos será la de migrar y depositarse en aquellas localizaciones en las que el proceso de resorción ósea se encuentre aumentado y en donde el osteoclasto como célula activa se encuentra degradando los componentes orgánicos y minerales del hueso (Ashcroft et al., 2003). La molécula de bifosfonato se deposita entre los cristales de hidroxiapatita, los cuáles serán disueltos por el osteoclasto, procediendo con ello a la endocitosis del bifosfonato. Una vez dentro, la molécula de bifosfonato se deposita en el citosol del osteoclasto, procediendo a su inactivación definitiva.

Son múltiples las causas por las que puede verse incrementado en cierta medida el metabolismo óseo. Entre ellas podemos establecer un ámplio número de patologías entre las que cobran especial relevancia los procesos neoplásicos entre los que destacan el cáncer de pulmón, de próstata, de seno, o la enfermedad de Pager (JiménezSorianao \& Bagan, 2005; Ruggiero et al., 2004).

\section{Caso clínico 1}

Paciente de 48 años de edad, no fumadora, menopáusica, con antecedente personal de hipotiroidismo, taquicardias paroxísticas supraventriculares desde 1996, alergia a tetraciclinas y amoxicilina clavulánica. Diagnosticada en 1996 de cáncer de mama y tratada con cirugía conservadora asociada a quimioterapia adyuvante (C.E.F - 60 x 7 ciclos), Radioterapia externa (Co 60 - 50 Gy) y hormonoterapia (Tamoxifen por 5 años). 
En remisión clínica hasta julio de 2005, fecha en que a consecuencia del estudio por parálisis del nervio recurrente izquierdo le fueron detectadas metástasis pulmonares múltiples, mediastínicas así como diversas letálides en cuero cabelludo.

Inició tratamiento quimioterápico en esquema (gemcitabina + vinorelbina $\times 7$ ciclos), que completó satisfactoriamente y bajo el cual desaparecieron las lesiones cutáneas. Los focos mediastínicos persistieron, para lo cual se instauró nueva línea de quimiopterapia con Taxol por 10 ciclos en monoterapia. El estudio de extensión (T.A.C), reveló múltiples lesiones en sendos campos pulmonares, así como implantes a nivel óseo con predominio en eje axial, a nivel de D4, D12 y L3, L5.

Se planteó esquema de quimioterapia con capecitabina por 8 ciclos, que completó con buena tolerancia. El estudio de extensión (P.E.T), evidenció la desaparición de las captaciones mediastínicas y pulmonares descritas en estudios previos, persistiendo las lesiones óseas a nivel vertebral.

Se decidió continuar tratamiento quimioterápico con fulvestran y bifosfonatos, (ácido zoledrónico, a dosis de 4 mg cada 28 días por vía intravenosa).

A fecha de octubre de 2007, la paciente consultó por dolor intenso en región mandibular izquierda, motivo por el que se canceló el tratamiento con Zometa ante la posible sospecha de osteonecrosis mandibular por bifosfonatos. La paciente fue derivada al servicio de cirugía maxilofacial, el cual ante la progresiva agudización del cuadro procedió a su ingreso. Mediente ortopantomografía, fue diagnosticada de osteonecrosis mandibular secundaria al tratamiento con bifosfonatos. La paciente recibió tratamiento con antibioterapia (clindamicina $300 \mathrm{mg}$ ), vía intravenosa, cada 8 horas y durante 15 días. La evolución fue favorable lográndose un correcto control del dolor en la zona así como la progresiva desaparición del foco infeccioso-lesivo mandibular.

\section{Caso clínico 2}

Paciente de 68 años de edad, no fumadora, hipertensa y con antecedente de quiste en mama derecha, intervenida en 1981 (tumorectomía + linfadenectomía axilar), con resultado anatomopatológico de mastopatía fibroquística.

En 1993 fue diagnosticada de cáncer de mama, siendo tratada con cirugía radical (mastectomía simple) y hormonoterapia (tamoxifen por 5 años), que finalizó en junio de 1998, con excelente tolerancia y respuesta al mismo.

La paciente, permaneció en remisión clínica hasta diciembre de 2002, fecha en la que se detectó una elevación en los marcadores tumorales (Ca 15.3: 215,50), motivo por el que se realizó una tomografía por emisión de positrones (P.E.T.). El resultado evidenció la presencia de múltiples implantes óseos metastásicos, a nivel de columna (regiónes dorsal: D8, D9 y lumbar: L5), así como en articulación sacro-ilíaca izquierda y escápula izquierda.

Se propuso tratamiento quimioterápico en régimen de (taxol + epirrubicina) asociado a bifosfonatos (ácido zoledrónico, a dosis de $4 \mathrm{mg}$, cada 28 días por vía intravenosa), completando un total de 14 ciclos con buena tolerancia. El estudio de extensión postquimioterapia, reveló la persistencia de las lesiones óseas metastásicas por lo que se decidió continuar con quimioterapia en esquema de Xeloda, acompañado de bifosfonatos (ácido zoledrónico, a dosis de $4 \mathrm{mg}$, cada 28 días por vía intravenosa), recibiendo un total de 19 ciclos, con una tolerancia aceptable. El estudio de extensión resultó negativo, pese a lo cual, se estimó conveniente continuase en tratamiento con ácido zoledrónico.

La paciente permaneció asintomática hasta abril de 2005, fecha en la que desarrolló un cuadro de trombosis venosa profunda (T.V.P), en sector ilio femoro-poplíteo izquierdo, por el que sería ingresada para tratamiento anticoagulante. La evolución resultó favorable.

En junio de 2005, acudió a nuestra unidad para revisión, realizándose un estudio de extensión el cual resultó positivo para metástasis óseas múltiples en sendas regiones costales, así como, 12 lesiones a nivel subpleural, sobre segmento posterior de lóbulo inferior izquierdo. Ante tal eventualidad se decidió tratamiento con Fulvestran cada 28 días, asociado con ácido zoledrónico a dosis de $4 \mathrm{mg}$, cada 28 días por vía intravenosa.

Con fecha de julio de 2007, la paciente manifestó sensación de quemazón y dolor en región mandibular izquierda, motivo por el que se canceló el tratamiento con ácido zoledrónico, ante la posible sospecha de osteonecrosis mandibular.

La paciente fue derivada al servicio de cirugía maxilofacial para valoración y posible tratamiento, siendo ingresada en dicho servicio para administración de tratamiento antibiótico (vía intravenosa). La evolución fue favorable. A continuación, dicho servicio indicó la necesidad de 
completar tratamiento con oxígeno en cámara hiperbárica, recibiendo dos ciclos del mismo con excelente respuesta.

A fecha 16 de noviembre de 2007, la paciente fue intervenida en el servicio de Cirugía Maxilofacial para legrado quirúrgico y secuestrectomía, con curso postoperatorio favorable. Actualmente, la paciente permanece estable clínicamente, sin hallazgos de interés y en tratamiento con fulvestran.

\section{Caso clínico 3}

Paciente de 59 años de edad, no fumadora, con antecedente personal de enfermedad de Graves Basedow. En 1988 fue diagnosticada de cáncer de mama, siendo tratada con cirugía radical (mastectomía radical modificada), con resultado anatomopatológico de carcinoma ductal infiltrante escirro, de moderada diferenciación celular, (grado II) según (Bloom - Richardson) y metástasis en 11 de las 21 adenopatías aisladas.

La paciente completó tratamiento con qluimioterapia adyuvante, en esquema (C.M.F) x 6 ciclos, con buena tolerancia, y radioterapia externa secuencial, con una dosis total de 48 Gy y excelente tolerancia. El estudio de extensión postquimioterapia (enero de 1988), resultó negativo.

En octubre de 1991, fue diagnosticada de macroadenoma hipofisario. Se practicó tratamiento quirúrgico, (craniotomía frontotemporal izquierda + hipofisectomía). El resultado anatomopatológico resultó ser positivo para adenoma. La paciente completó tratamiento con radioterapia externa, recibiendo una dosis total de 50 Gy, acompañado de tratamiento sustitutivo y tratamiento antitiroideo. La paciente, permaneció estable clínicamente hasta mayo de 1997, fecha en la que le fue detectada una metástasis ósea solitaria en cresta ilíaca izquierda. Se propuso tratamiento con radioterapia y quimioterapia secuenciales, en esquema (epirrubicina + taxol) x 6 ciclos, con tolerancia aceptable. El estudio de extensión postquimioterapia (octubre de 1997) fue negativo. Se decidió completar tratamiento con hormonoterapia en régimen de Tamoxifen $20 \mathrm{mg}$.

Permaneció en remisión clínica hasta septiembre del 2000, fecha en la que fueron detectadas metástasis pulmonares, para lo que se decidió continuar con hormonoterapia (aromasil), tratamiento que recibió hasta enero de 2002, fecha en la que se suspendió por progresión de la enfermedad a nivel óseo. Se propuso continuar con quimioterapia en esquema de (herceptín + cisplatino), del que completó 8 ciclos presentando una gran toxicidad. Se estableció una nueva línea de tratamiento quimioterápico con capecitabina, de la que sólo llegó a recibir un ciclo por intolerancia. El estudio de extensión (julio de 2002), manifestó un estado estacionario de las lesiones óseas.

En septiembre de 2002, fue diagnosticada de Paget vulvar, para el cual recibió tratamiento quirúrgico (vulvectomía). La paciente completó tratamiento con quimioterapia en esquema de Trastuzumab, acompañado de bifosfonatos (ácido zoledrónico, a dosis de $4 \mathrm{mg}$ cada 28 días por vía intravenosa), tratamiento que completó hasta mayo de 2003, fecha en la que se produjo un nuevo avance de las lesiones óseas. Se instaura nueva línea de tratamiento con (UTEFOS) x 6 ciclos, hasta diciembre de 2003.

En Enero de 2004, se modificó el tratamiento pasando a una nueva línea con Femara asociado al tratamiento con pamidronato que aun no había cesado, prolongándose dicho esquema hasta mayo de ese mismo año, fecha en la que se desestimaría continuar dada la progresión general de su enfermedad (metástasis óseas y hepática única). Se practicó hepatectomía (julio de 2005), con resultado y evolución favorable.

Tras cirugía hepática se decidió continuar con quimioterapia (herceptín + fulvestran + pamidronato), tratamiento del que sólo llegó a administrarse tres ciclos. A continuación, comenzó con una nueva línea (taxol + herceptín + faslodex), tratamiento que recibiría hasta abril de 2006, fecha en la que la paciente manifiestó dolor intenso en región maxilar izquierda con extensión hacia rama mandibular del mismo lado, motivo por el cual sería suspendido el tratamiento con ácido zoledrónico, ante la posible sospecha de osteonecrosis maxilo-mandibular. La paciente fue derivada al servicio de cirugía maxilofacial donde se la diagnosticó de osteomielitis secundaria al tratamiento con bifosfonatos y tratada con antibioterapia y antiálgicos.

En octubre de 2007, decidió trasladarse de hospital para recibir tratamiento con oxígeno hiperbárico en las lesiones maxilar y mandibular. La mejoría en tales lesiones resultó no ser sustancial. A fecha de diciembre de 2007, y a causa de la intensa algia provocada por las lesiones necróticas maxilares y mandibulares se consideró la posibilidad de intervenir quirúrgicamente las mismas, posibilidad que quedó desestimada dado el mal estado general de la paciente y progresión generalizada de su enfermedad.

Con fecha de febrero de 2007, la paciente ingresó por estado estuporoso con varios días de evolución, cuadro que se prolongaría en los sucesivos días de su hospitalización, culminando con su fallecimiento una semana más tarde. 


\section{DISCUSIÓN}

Los bifosfonatos constituyen una herramienta fundamental en el manejo de las lesiones secundarias a patologías tan complejas como son las metástasis óseas, en el caso de ciertos tipos de cáncer (Powles et al., 2006; Hirbe et al., 2006).

Su frecuente uso en la terapéutica del cáncer durante los últimos años, ha provocado un repunte en el número de pacientes que asociado a su tratamiento con bifosfonatos presentan cuadros de osteonecrosis avascular en las regiones maxilar y mandibular (Merigo et al., 2006).

Sin embargo, conviene destacar el efecto positivo que el tratamiento con bifosfonatos reporta para los pacientes respecto a su calidad de vida y pronóstico en enfermedades complejas como es el caso que nos ocupa (el cáncer) (Gnant et al., 2009; Brufsky et al., 2008). La posibilidad de desarrollar una complicación del tipo de la necrosis avascular maxilo-mandibular, no debe por otra parte ser un factor de- cisorio para administrar o no bifosfonatos. Ahora bien, la detección y diagnóstico de lesiones de este tipo habrán de ir asociadas necesariamente al cese inmediato del bifosfonato y al inicio de una correcta higiene en región oral, utilizando solución de clorhexidina al $0,12 \%$, así como una correcta cobertura antibiótica para evitar la aparición de focos infecciosos recidivantes (Saad et al., 2007).

Por su parte, la eficacia del tratamiento con oxigeno mediante cámara hiperbárica, para mejorar la oxigenación local de las zonas afectas, constituye un hecho a demostrar aun en la actualidad (Jaimes et al., 2008).

Es a nuestro juicio necesario, ser perseverantes en el estudio de esta entidad patológica, siendo fundamental el análisis profundo de su etiopatogenia, así como también su diagnóstico y tratamiento precoz.

AGRADECIMIENTOS: Damos las gracias a las pacientes y sus familias por la colaboración en todo momento prestada para la realización de este trabajo.

GONZÁLEZ, J. E.; ÁLVAREZ, F. J. \& AGUILAR C. M. J. Mandibular and maxilar osteonecrosis secondary to bisphosphonates. Introduction of three new cases. Int. J. Morphol., 29(3):1022-1027, 2011.

SUMMARY: The use of bisphosphonates is now particularly important in the therapeutic management of various pathologies, as an integral part of standard therapy. However, in an almost parallel form, a large number of cases have been documented in which the patient following treatment with bisphosphonates, developed a vascular necrosis event in the maxillary and/or mandibular region. This is an event of complex approach which tends to compromise and undermine the quality of life for our patients. However the possible occurrence of this complication should not on the other hand, imply misgivings about their use in therapy, but rather constitute an event to be monitored during the time of therapy. The aim of this paper is to advise about possible complications in patients receiving bisphosphonate therapy through literature review and the presentation of these three new clinical cases.

KEY WORDS: Bisphosphonate; Osteonecrosis, Cancer.

\section{REFERENCIAS BIBLIOGRÁFICAS}

Ashcroft, J.; Davies, E. \& Morgan, J. Aetiology of bone disease and the role of bisphosphonates in multiple myeloma. Lancet Oncol., 4(5):284-92, 2003.

Bagan, J. V.; Jimenez, Y.; Murillo, J.; Hernandez, S.; Poveda, R.; Sanchis, J. M.; Diaz, J. M. \& Scully, C. Jaw osteonecrosis associated with bisphosphonates: multiple exposed areas and its relationship to teeth extractions. Study of 20 cases. Oral Oncol., 42(3):327-9, 2006.

Brufsky, A.; Bundred, N.; Coleman, R.; Lambert-Falls, R.; Mena, R.; Hadji, P.; Jin, L.; Schenk, N.; Ericson, S.; Perez, E. A.; Z-FAST \& ZO-FAST Study Groups. Integrated analysis of zoledronic acid for prevention of aromatase inhibitor associated bone loss in postmenopausal women with early breast cancer receiving adjuvant letrozol. Oncologist, 13:503-14, 2008.
Coleman, R. E.; Body, J. J.; Gralow, J. R. \& Lipton, A. Bone loss in patients with breast cancer receiving aromatase inhibitors and associated treatment strategies. Cancer Treat. Rev., 34:S31-42, 2008.

Dimitrakopoulos, I.; Magopoulos, C. \& Karakasis, D. Biphosphonates-induced avascular osteonecrosis of the jaws: a clinical report of 11 cases. Int. J. Oral Maxillofac. Surg., 35:588-93, 2006.

Durie, B. G.; Katz, M. \& Crowley, J. Osteonecrosis of the jaw and bisphosphonates. N. Engl. J. Med., 353:99-102, 2005a.

Durie, B.G.M.; Katz, M.; MacCoy, J.; Crowley, J. Osteonecrosis of the jaws in myeloma: analysis of risk factors including time dependency of Aredia and Zometa use, steroid use and underlying dental problems. Haematologica / Hem Journal, 90(S1):190, 2005 b.

Gnant, M.; Mlineritsch, B.; Schippinger, W.; Luschin- 
Ebengreuth, G.; Pöstlberger, S.; Menzel, C.; Jakesz, R.; Seifert, M.; Hubalek, M.; Bjelic-Radisic, V.; Samonigg, H.; Tausch, C.; Eidtmann, H.; Steger, G.; Kwasny, W.; Dubsky, P.; Fridrik, M.; Fitzal, F.; Stierer, M.; Rücklinger, E.; Greil, R.; ABCSG-12 Trial Investigators, Marth, C. Endocrine therapy plus zoledronic acid in premenopausal breast cancer. N. Engl. J. Med., 360:679-91, 2009.

Gutta, R. \& Louis, R.J. Bisphosphonates and osteonecrosis of the jaws: Science and rationale. Oral Surg. Oral Med. Oral Pathol. Oral Radiol. Endod., 104(2):186-93, 2007.

Guirado, M.; Gálvez, E. \& Rodríguez-Lescure, A. ¿Qué papel pueden representar los bifosfonatos en el tratamiento adyuvante del cáncer de mama? Rev. Cancer, 23:26-8, 2009.

Hirbe, A.; Morgan, E. A.; Uluçkan, Ö. \& Weilbaecher, K. Skeletal complications of breast cancer therapies. Clin. Cancer Res., 12:6309-14, 2006.

Jaimes, M.; Chaves Netto, H. D. M.; Olate, S.; Chaves, M. M. G. A. \& Barbosa, A. J. R. Bifosfonatos y osteonecrosis de los maxilares. Consideraciones sobre su tratamiento. Int. J. Morphol., 26(3):681-8, 2008.

Jiménez-Soriano, Y. \& Bagan, J. V. Bisphosphonates, as a new cause of drug - induced jaw osteonecrosis: an update. Med. Oral Patol. Oral Cir. Bucal. 1(10)Suppl., 2:88-91, 2005.

Marx, R. E. Pamidronate (Aredia) and zoledronate (Zometa) induced avascular necrosis of the jaws: a growing epidemic. J. Oral Maxillofac. Surg., 61(10):1115-7, 2003.

Marx, R. E.; Sawatari, Y.; Fortin, M. \& Broumand, V. Bisphosphonate-induced exposed bone (osteonecrosis/ osteopetrosis) of the jaws: risk factors, recognition, prevention, and treatment. J. Oral Maxillofac. Surg., 63(11):1567-75, 2005.

Merigo, E.; Manfredi, M.; Meleti, M.; Guidotti, R.; Ripasarti, A.; Zanzucchi, E.; D'Aleo, P.; Corradi, D.; Corcione, L.; Sesenna, E.; Ferrari, S.; Poli, T.; Bonaninil, M. \& Vescovi, P. Bone necrosis of the jaws associated with bisphosphonate treatment: a report of twenty-nine cases. Acta Biomed., 77(2):109-17, 2006.

Naveau, A. \& Naveau, B. Osteonecrosis of the jaw in patients taking bisphosphonates. Joint Bone Spine., 73(1):7-9, 2006.

Lenz, J. H.; Steiner-Krammer, B.; Schmidt, W.; Fietkau, R.; Mueller, P. C. \& Gundlach, K. K. Does a vascular necrosis of the jaws in cancer patients only occur following treatment with bisphosphonates? J. Craniomaxillofac.
Surg., 33(6):395-403, 2005.

Lipton, A.; Cook, R.; Saad, F.; Major, P.; Garnero, P.; Terpos, E.; Brown, J. E. \& Coleman, R. E. Normalization of bone markers is associated with improved survival in patients with bone metastases from solid tumors and elevated bone resorption receiving zoledronic acid. Cancer, 113:193-201, 2008.

Powles, T.; McCroskey, E. \& Paterson, A. Oral bisphosphonates as adjuvant therapy for operable breast cancer. Clin. Cancer Res., 12: 6301s-4s, 2006.

Rogers, M. J. From molds and macrophages to mevalonate: a decade of progress in understanding the molecular mode of action of bisphosphonates. Calcif. Tissue Int., 75:45161, 2004.

Ruggiero, S. L.; Bhoomi, M.; Rosemberg, T. \& Engroff, S. L. Osteonecrosis of the jaws associated with the use of biphosphonates: a review of 63 cases. J. Oral Maxillofac. Surg., 62:527-34, 2004.

Saad, F.; Lipton, A.; Cook, R.; Chen, Y. M.; Smith, M. \& Coleman, R. Pathological fractures correlate with reduced survival in patients with malignant bone disease. Cancer, 110:1860-7, 2007.

Santini, D.; Vicenzi, B.; Avvisati, G.; Dicuonzo, G.; Battistoni, F.; Gavasci, M.; Salerno, A.; Denaro, V. \& Tonini, G. Pamidronate induces modifications of circulating angiogenic factors in cancer patients. Clin. Cancer Res., 8:1080-4, 2002.

Thakkar, S. G.; Isada, C.; Smith, J.; Karam, M. A.; Reed, J.; Tomford, J. W.; Englund, K.; Richmond, M.; Licata, A.; Hatch, C. \& Hussein, M. A. Jaw complications associated with bisphosphonate use in patients with plasma cell dyscrasias. Med. Oncol., 23(1):51-6, 2006.

Vitté, C.; Fleisch, H. \& Guenther, H.L. Bisphosphonates induce osteoblasts to secrete an inhibitor of osteoclastmediated resorption. Endocrinology, 137:2324-33, 1996.

Dirección para correspondencia:

Dr. Emilio González Jiménez

Departamento de Enfermería

Facultad de Ciencias de la Salud

Universidad de Granada

Avenida de Madrid s/n

C. P: (18071)

Granada - ESPAÑA

Recibido : 06-01-2011

E-mail: emigoji@ugr.es
Aceptado: 22-06-2011 